\title{
Persistence for a Two-Stage Reaction-Diffusion System
}

\author{
Robert Stephen Cantrell ${ }^{1, *}$, Chris Cosner ${ }^{1}$ and Salomé Martínez ${ }^{2}$ \\ 1 Department of Mathematics, University of Miami, Coral Gables, FL 33124, USA; gcc@math.miami.edu \\ 2 Departamento de Ingeniería Matemática and Centro de Modelamiento Matemático, \\ UMI 2807 CNRS-UChile, Universidad de Chile, 8370456 Santiago, CHILE; samartin@dim.uchile.cl \\ * Correspondence: rsc@math.miami.edu
}

Received: 3 February 2020; Accepted: 5 March 2020; Published: 11 March 2020

\begin{abstract}
In this article, we study how the rates of diffusion in a reaction-diffusion model for a stage structured population in a heterogeneous environment affect the model's predictions of persistence or extinction for the population. In the case of a population without stage structure, faster diffusion is typically detrimental. In contrast to that, we find that, in a stage structured population, it can be either detrimental or helpful. If the regions where adults can reproduce are the same as those where juveniles can mature, typically slower diffusion will be favored, but if those regions are separated, then faster diffusion may be favored. Our analysis consists primarily of estimates of principal eigenvalues of the linearized system around $(0,0)$ and results on their asymptotic behavior for large or small diffusion rates. The model we study is not in general a cooperative system, but if adults only compete with other adults and juveniles with other juveniles, then it is. In that case, the general theory of cooperative systems implies that, when the model predicts persistence, it has a unique positive equilibrium. We derive some results on the asymptotic behavior of the positive equilibrium for small diffusion and for large adult reproductive rates in that case.
\end{abstract}

Keywords: reaction-diffusion; spatial ecology; population dynamics; stage structure; dispersal

AMS Classification: 92D40; 92D50; 35P15; 35K57

\section{Introduction}

The question of how dispersal interacts with spatial heterogeneity to influence population dynamics and species interactions has been studied extensively in recent years, specifically from the viewpoint of reaction-diffusion systems and related models-see, for example, [1-3] and the references cited therein. Most work on that topic assumes that each population is structured only by space and has only one mode of dispersal. However, populations are often structured by age, stage, or other attributes, and there may be variation among individuals in their dispersal rates or patterns. Here we will examine how the presence of a stage structure influences how diffusion rates influence population dynamics in a class of reaction-diffusion models for a population with two stages. In the case of a population with logistic growth, without age or stage structure, diffusing in a closed bounded spatially heterogeneous environment that is constant in time, it is well known that reaction-diffusion models predict that slower diffusion rates are advantageous relative to faster diffusion-see [4,5]. The results in [5] also hold for patch models. More broadly, a wide class of models arising in population genetics, population dynamics, and related areas display some version of the reduction principle, which says that dispersal, which causes faster mixing, typically reduces the rate of population growth-see [6]. However, the situation seems to be quite different in the case of stage structured populations. In [7], the authors considered a discrete-time patch model for a structured population and found that, in some cases, there was no selection against faster dispersal. The goal of the present paper is to use a spatially explicit reaction-diffusion model to understand how the spatial 
distributions of habitats that are favorable for reproduction by adults and those that are favorable for survival and growth by juveniles affect whether faster diffusion is advantageous or harmful for a stage structured population. We will see that the answer depends on the details of the spatial distribution of favorable and unfavorable habitats.

The type of reaction-diffusion model we will study is

$$
\left\{\begin{array}{l}
\frac{\partial u}{\partial t}=d_{1} \Delta u+r(x) v-s(x) u-a(x) u-b(x) u^{2}-c(x) u v \text { in } \Omega, t>0 \\
\frac{\partial v}{\partial t}=d_{2} \Delta v+s(x) u-e(x) v-f(x) v^{2}-g(x) u v \text { in } \Omega, t>0 \\
\nabla u \cdot v=\nabla v \cdot v=0 \text { on } \partial \Omega, t>0 .
\end{array}\right.
$$

$\Omega$ is a bounded domain in $\mathbb{R}^{N}$, and $v$ is the outward unit normal to $\partial \Omega$, such that the system has Neumann boundary conditions, which are the no-flux boundary conditions for simple diffusion. In this system, $u$ and $v$ represent the population densities of juveniles and individuals that have reached reproductive age, i.e. adults, respectively, of the same species. Thus, the term $s(x)$ represents the rate at which juveniles mature into adults, which is determined by the fraction of individuals that reach reproductive age and the rate at which they mature, while $r(x)$ accounts for the local fecundity of adults such that $r(x) v(x)$ describes that rate at which new juveniles are produced by an adult population with density $v$ at location $x$. The terms $a(x), b(x), c(x), e(x), f(x)$, and $g(x)$ account for per-capita death rates and saturation factors due to logistic self-limitation. The diffusion coefficients $d_{1}$ and $d_{2}$ account for the the dispersal rates of juveniles and adults, respectively. The coefficients are all assumed to be nonnegative and continuous in $\bar{\Omega}$. This is the type of model for a stage structured population introduced in [8]. Related models with a different interpretation are discussed in [9,10] and in the references in those papers. The model expressed in Equation (1) is not an explicitly age-structured model. It assumes that individuals in the juvenile stage mature at some spatially dependent rate but does not track the age of individuals within each stage. Explicitly age structured models are considered in [11-13]. A different way of modeling an age-structured population, based on delayed reaction diffusion equations, is developed in [14]. Our focus here is on how spatial heterogeneity, dispersal, and stage structure interact, so we chose to use the simplest possible formulation of stage structure. In the case where $c=g=0$, the system is cooperative, and the methods and results of $[15,16]$ would apply to it. The linearization of Equation (1) around $(0,0)$ is cooperative, so the results of [15] apply to it; in particular, with a few technical assumptions, they imply that it has a principal eigenvalue.

The main questions we will address in this work are related to understanding the roles of the different functions and coefficients in Equation (1) in the persistence of the species. For the remainder of the paper we will focus primarily on understanding how the principal eigenvalue of the linearization of Equation (1) around $(0,0)$ depends on the coefficients and what that dependence means biologically. We will see that whether faster diffusion is harmful or helpful for the persistence of the population depends on the details of the distribution of habitats that are favorable for adult reproduction and those that are favorable for juvenile survival and maturation. In some cases slower diffusion is still an advantage, but sometimes faster diffusion turns out to be helpful, and sufficiently fast diffusion may even be necessary for persistence. The spatial distribution of habitats favorable to adult reproduction $(r(x)$ large) relative to those favorable to juvenile development $(s(x)$ large) turns out to be important in some cases. Our analysis here is similar in spirit to the sorts of results obtained for diffusive Lotka-Volterra competition models in [3,17-19]. In particular, we will examine the behavior of the system for small, large, and general diffusion rates. Related results for some epidemiological models are derived in $[20,21]$.

The linearization of Equation (1) around $(0,0)$ has a principal eigenvalue whose sign determines whether the model predicts persistence or extinction. Since the sign of the principal eigenvalue of 
the linearization of Equation (1) around $(0,0)$ determines the fate that Equation (1) predicts for the population it describes, we will study in detail the following problem:

$$
\left\{\begin{aligned}
d_{1} \Delta \varphi+r(x) \psi-(s(x)+a(x)) \varphi & =\lambda \varphi \text { in } \Omega \\
d_{2} \Delta \psi+s(x) \varphi-e(x) \psi & =\lambda \psi \text { in } \Omega \\
\nabla \varphi \cdot v=\nabla \psi \cdot v & =0 \text { on } \partial \Omega
\end{aligned}\right.
$$

\section{Basic Properties}

In this section, we discuss some basic properties of Equation (1). From now on, we assume that $r, s, a, b, c, e, f, g \in C^{\alpha}(\bar{\Omega}), \partial \Omega$ is of class $C^{2, \alpha}$, and that the following hypotheses hold:

Hypothesis 1 (H1). $r(x), s(x) \geq 0$ in $\Omega$, with $r\left(x_{r}\right) \neq 0, s\left(x_{s}\right) \neq 0$ for some $x_{r}, x_{s} \in \Omega$.

Hypothesis 2 (H2). $a(x), c(x), e(x), g(x) \geq 0$ in $\Omega$.

Hypothesis 3 (H3). $b(x)>0, f(x)>0$ in $\bar{\Omega}$.

The model expressed in Equation (1) has many mathematical features in common with the models discussed in $[9,10]$ for populations where individuals can switch between two different movement modes. A key feature is that the linear part of Equation (1) is cooperative, so it will have a principal eigenvalue which determines the stability of the equilibrium $(0,0)$ and hence the persistence or extinction of the population. Another key feature of Equation (1) is that the nonlinearity is subhomogeneous. The maximum principle and existence of principal eigenvalues for cooperative linear systems such as the linear part of the right side of Equation (1) are derived in [15]. The general theory for systems such as Equation (1) is developed in [16] for the fully cooperative case (where $c=g=0$, such that adults only compete with other adults and juveniles with other juveniles) and in the general case in $[8-10,22]$. As expected, the sign of the principal eigenvalue of the linearization of Equation (1) around $(0,0)$ gives us the relevant information to study the persistence of the species. If it is positive, the population will persist. If it is nonpositive, the population will go extinct. In the case where the coefficients of $c$ and $g$ in Equation (1) are zero such that the system is cooperative, the results and methods of [16] imply that, if the principal eigenvalue of the linear part is positive, then the system has a unique globally attractive equilibrium. If those coefficients are small, the methods of $[9,10]$ can be applied to show that Equation (1) is asymptotically cooperative, and still has a unique globally attractive positive equilibrium. Combining results that are given in $[8-10,15,16]$ or that follow directly by the same arguments used in those papers, we have the following:

Lemma 1. The eigenvalue problem expressed in Equation (2) has a unique principal eigenvalue $\lambda_{1}$ that is characterized by having a positive eigenvector $(\varphi, \psi)$.

Lemma 2. If $\lambda_{1}>0$, then the system expressed in Equation (1) is persistent and has at least one positive equilibrium. If $\lambda_{1} \leq 0$, then $(0,0)$ is globally asymptotically stable in Equation (1).

Lemma 3. If $\lambda_{1}>0$ and $c$ and $g$ are sufficiently small, then the system expressed in Equation (1) has a unique globally attracting positive equilibrium.

Remark 1. In the case that $c=g=0$, the system expressed in Equation (1) is cooperative and hence generates a monotone semi-flow on appropriate spaces. 


\section{The Case of $d_{1}, d_{2}$ Small}

Following the approach in [23], we will establish the asymptotic behavior of the principal eigenvalue of Equation (2) when $d_{1}, d_{2}$ are small and, in the fully cooperative case (where $c \equiv g \equiv 0$ ), the profile of the nonnegative solutions of the corresponding steady state system for Equation (1).

$$
\left\{\begin{array}{l}
d_{1} \Delta u+r(x) v-s(x) u-a(x) u-b(x) u^{2}=0 \text { in } \Omega, \\
d_{2} \Delta v+s(x) u-e(x) v-f(x) v^{2}=0 \text { in } \Omega, \\
\nabla u \cdot v=\nabla v \cdot v=0 \text { on } \partial \Omega
\end{array}\right.
$$

as well. Related results are derived in [16]. We observe that the associated kinetic system, which corresponds to Equation (1), is given by

$$
\left\{\begin{array}{l}
u_{t}=r(x) V(x)-s(x) U(x)-a(x) U(x)-b(x) U^{2}(x)-c(x) U(x) V(x)=0 \\
v_{t}=s(x) U(x)-e(x) V(x)-f(x) V^{2}(x)-g(x) U(x) V(x)=0
\end{array}\right.
$$

for each $x \in \Omega$.

For each $x$, this system shares the same properties as Equation (1) given in Lemmas 1-3, which we state for convenience.

Lemma 4. Set $x \in \Omega$. The linearization around $(0,0)$ of Equation (4) has a principal eigenvalue $\lambda_{1}(x)$. Moreover,

(i) If $\lambda_{1}(x) \leq 0$, then all the solutions with nonnegative initial condition of Equation (4) converge to $(0,0)$ when $t \rightarrow \infty$.

(ii) If $\lambda_{1}(x)>0$, then Equation (4) is persistent and has at least one positive equilibrium.

(iii) If $c \equiv g \equiv 0$ and $\lambda_{1}(x)>0$, then Equation (4) is cooperative and admits a unique positive equilibrium, which is the global attractor for all nonnegative, non-trivial solutions.

Observe that, when $d_{1}=d_{2}=0$, the eigenvalues of the linearization around $(0,0)$ of Equation (4) are the roots of $\operatorname{det}(A(x)-\lambda I)$, with

$$
A(x)=\left[\begin{array}{cc}
-(s(x)+a(x)) & r(x) \\
s(x) & -e(x)
\end{array}\right]
$$

By a simple computation, we obtain that the maximum eigenvalue is given by

$$
\Lambda(x)=\frac{1}{2}\left[-(s(x)+a(x)+e(x))+\sqrt{(s(x)+a(x)-e(x))^{2}+4 r(x) s(x)}\right],
$$

which is positive provided that $(s(x)+a(x)) e(x)-r(x) s(x)<0$. Our first result, which is a direct application of Theorem 1.4 of [23] (Theorem A1 in the Appendix A), states that this is indeed the necessary and sufficient condition to have a positive principal eigenvalue when $d_{1}$ and $d_{2}$ are small.

Proposition 1. The principal eigenvalue $\lambda_{1}$ of Equation (2) satisfies

$$
\lambda_{1} \rightarrow \max _{x \in \bar{\Omega}} \Lambda(x) \text { as } d_{1}, d_{2} \rightarrow 0
$$

Thus, there exists a $\delta>0$ such that if

$$
\min _{x \in \bar{\Omega}}((s(x)+a(x)) e(x)-r(x) s(x))<0,
$$


the principal eigenvalue of Equation (2) is positive for all $0<d_{1}, d_{2}<\delta$, while if

$$
\min _{x \in \bar{\Omega}}((s(x)+a(x)) e(x)-r(x) s(x))>0,
$$

the principal eigenvalue is negative.

As a consequence of this result, if Equation (9) holds, the unique nonnegative equilibrium of the system expressed in Equation (3) is $(0,0)$, and that equilibrium is globally attracting, whenever $d_{1}, d_{2}$ are small; if Equation (8) holds, the system expressed in Equation (3) is persistent, and has a positive equilibrium for small $d_{1}, d_{2}$. If Equation (8) holds and $c \equiv g \equiv 0$, then by Lemma 3 the system expressed in Equation (3) has a unique globally attracting positive equilibrium, which we denote by $\left(u_{d}, v_{d}\right)$ with $d=\left(d_{1}, d_{2}\right)$.

Throughout the remainder of this section, we will assume that $c \equiv g \equiv 0$ in $\Omega$, in which case, the system expressed in Equation (1) is cooperative.

The next result establishes the convergence of $\left(u_{d}, v_{d}\right)$ to the unique nonnegative steady state $(U(x), V(x))$ of the kinetic system, which satisfies

$$
\begin{aligned}
& (U(x), V(x)) \text { is positive where }(s(x)+a(x)) e(x)-r(x) s(x)<0, \\
& (U(x), V(x))=0 \text { where }(s(x)+a(x)) e(x)-r(x) s(x) \geq 0 .
\end{aligned}
$$

Theorem 1. Suppose that Equation (8) holds. Then $\left(u_{d}, v_{d}\right) \rightarrow(U, V)$ as $d \rightarrow 0$ locally uniformly in $\Omega$.

To prove this theorem, we follow the proof of Theorem 1.5 of [23], specifically their Proposition 5.2 in [23] and its hypotheses, which are listed in [23] as (A1)-(A4) and are given in the Appendix A as (L1)-(L4) to avoid confusion with the equation labels there. We should point out that Assumptions (A2) and (A3) of [23] do not hold in our case, so we cannot apply that result directly. The difference is that we allow situations where the kinetic system expressed in Equation (4) has a positive equilibrium for some values of $x \in \bar{\Omega}$ but not for others, whereas Condition (A2) requires a positive equilibrium for the kinetic system for all $x$. For that reason, we need to construct a version of the arguments in [23] that is local in $x$. Condition (A3) in [23] is used only to prove the existence of a nontrivial subsolution for a system corresponding to Equation (3), which is independent of $d_{1}, d_{2}$. We show the existence of the analogous local subsolutions we need in our case in the next lemma.

Lemma 5. Suppose that $\tilde{x} \in \Omega_{0}$, where

$$
\Omega_{0}=\{x \in \Omega /(s(x)+a(x)) e(x)-r(x) s(x)<0\} .
$$

Then there exists $d_{0}>0, \rho_{0}>0$, and a function $\underline{w}^{0}>0$ in $B(\tilde{x}, \rho) \subset \Omega_{0}$, which is a subsolution of Equation (3) for all $0<d_{1}, d_{2}<d_{0}$.

Proof. Let $p=\left(p_{1}, p_{2}\right)$, a positive eigenvector of $A(\tilde{x})$ with $p_{1}+p_{2}=1$, associated with its principal eigenvalue $\tilde{\sigma}>0$. Set $\varepsilon>0$ and small. We can choose $\rho>0$ such that $\overline{B(\tilde{x}, \rho)} \subset \Omega_{0}$ and

$$
\begin{aligned}
& |a(x)-\tilde{a}|<\varepsilon,|r(x)-\tilde{r}|<\varepsilon, \\
& |s(x)-\tilde{s}|<\varepsilon \text { and }|e(x)-\tilde{e}|<\varepsilon \text { for all } x \text { in } \overline{B(\tilde{x}, \rho)},
\end{aligned}
$$

where $\tilde{a}=a(\tilde{x}), \tilde{r}=r(\tilde{x}), \tilde{s}=s(\tilde{x})$, and $\tilde{e}=e(\tilde{x})$. Set $\eta>0$ as the principal eigenfunction associated with $\lambda>0$, the principal eigenvalue of

$$
\Delta \eta+\lambda \eta=0 \text { in } B(\tilde{x}, \rho), \eta=0 \text { on } \partial B(\tilde{x}, \rho),
$$


with $\max _{B(\tilde{x}, \rho)} \eta=1$. We claim that we can choose $\delta, \varepsilon, \rho, d_{0}>0$ such that $\delta \eta p$ is a subsolution of Equation (3) for all $d_{1}, d_{2}<d_{0}$. For simplicity and to keep the notation consistent with that in [23], we define $F(x, u, v)=\left(F_{1}(x, u, v), F_{2}(x, u, v)\right)$, with

$$
F_{1}(x, u, v)=r v-s u-a u-b u^{2} \text { and } F_{2}(x, u, v)=s u-e v-f v^{2}=0,
$$

where we have omitted the variable $x$ in $a, b, e, r, s, f$ to shorten the expressions. Observe that

$$
\begin{aligned}
& F_{1}(x, \delta \eta p)=\delta \eta\left(\tilde{\sigma} p_{1}+(r-\tilde{r}) p_{2}-(s-\tilde{s}) p_{1}-(a-\tilde{a}) p_{1}-b \delta p_{1}^{2} \eta\right) \\
& F_{2}(x, \delta \eta p)=\delta \eta\left(\tilde{\sigma} p_{2}+(s-\tilde{s}) p_{1}-(e-\tilde{e}) p_{2}-f \delta p_{2}^{2} \eta\right)
\end{aligned}
$$

and using Equation (12), we obtain that, if we choose $\varepsilon>0$ and a small $\delta$, we have that

$$
\begin{aligned}
& F_{1}(x, \delta \eta p) \geq \delta \eta\left(\tilde{\sigma} p_{1}-\varepsilon p_{2}-2 \varepsilon p_{1}-b \delta p_{1}^{2} \eta\right)>\delta \eta \frac{\tilde{\sigma}}{2} p_{1} \\
& F_{2}(x, \delta \eta p) \geq \delta \eta\left(\tilde{\sigma} p_{2}-\varepsilon p_{1}-\varepsilon p_{2}-f \delta p_{2}^{2}\right)>\delta \eta \frac{\tilde{\sigma}}{2} p_{2} .
\end{aligned}
$$

Therefore, replacing these inequalities in Equation (3), we obtain

$$
\begin{aligned}
& d_{1} \delta p_{1} \Delta \eta+F_{1}(x, \delta \eta p) \geq \delta \eta\left(-d_{1} p_{1} \lambda+\frac{\tilde{\sigma}}{2} p_{1}\right) \\
& d_{2} \delta p_{2} \Delta \eta+F_{2}(x, \delta \eta p) \geq \delta \eta\left(-d_{2} p_{2} \lambda+\frac{\tilde{\sigma}}{2} p_{2}\right)
\end{aligned}
$$

hence, if we set $d_{0}=\frac{\tilde{\sigma}}{2 \lambda}$. we obtain the desired result.

Using this lemma we can follow the proof of Proposition 5.2 in [23]. To facilitate our exposition, we will use the same notation. Set the operators $D=\operatorname{diag}\left(d_{1}, d_{2}\right), \mathcal{L}=\operatorname{diag}(\Delta, \Delta)$. To prove Theorem 1 , we will state the needed lemmas, discussing their relationships with the lemmas in [23] leading to the proof of Proposition 5.2.

Suppose that Equation (8) holds, setting $\underline{w}^{0}=\eta \delta p$ as in Lemma 5, and $\bar{w}^{0}=M$ where $M>0$ is given in Assumption (A4) such that $F_{1}(x, u, v) \leq-c u$ and $F_{2}(x, u, v) \leq-c v$ for all $u, v \geq M$ and $x \in \Omega$, with $c>0$ fixed. Set $K>0$ such that $K+\partial_{u} F_{1}(x, u, v)>0$ and $K+\partial_{v} F_{2}(x, u, v)>0$ for all $0 \leq u, v \leq M$, and we define $z=\bar{w}^{k}$ as the unique solution of

$$
\left\{\begin{array}{l}
-D \mathcal{L} z+K z=K u+F(x, u) \text { in } \Omega, \\
\nabla z \cdot v=0 \text { on } \partial \Omega,
\end{array}\right.
$$

for $u=\bar{w}^{k-1}$.

Lemma 6. Suppose that Equation (8) holds. For every $k$, we have $\underline{w}^{0}<\bar{w}^{k+1}<\bar{w}^{k}<\bar{w}^{0}$, and as $k \rightarrow \infty, \bar{w}^{k}$ converges uniformly to the unique positive solution $w$ of Equation (3), which satisfies $\underline{w}^{0}<w<\bar{w}^{k}$ in $\Omega$ for all $k \geq 0$.

Proof. We will prove that $\underline{w}^{0}<\bar{w}^{k}$ by induction. Suppose this is true for $k$. Observe that $\underline{w}^{0}<\bar{w}^{0}$ by construction. In the set, $B(\tilde{x}, \rho) \subset \Omega_{0}$ as in Lemma $5 \bar{w}^{k+1}$ satisfies

$$
-D \mathcal{L}\left(\bar{w}^{k+1}-\underline{w}^{0}\right)+K\left(\bar{w}^{k+1}-\underline{w}^{0}\right)=K\left(\bar{w}^{k}-\underline{w}^{0}\right)+F\left(x, \bar{w}^{k}\right)-F\left(\underline{w}^{0}\right),
$$


in $B(\tilde{x}, \rho)$. By the induction hypothesis $\underline{w}^{0}<\bar{w}^{k}$, whence $K \bar{w}^{k}+F\left(x, \bar{w}^{k}\right)>0$; hence, by the strong maximum principle applied to each component, we have that $\bar{w}^{k+1}>0$ in $\bar{\Omega}$. Thus, we have

$$
\left\{\begin{array}{l}
-D \mathcal{L}\left(\bar{w}^{k+1}-\underline{w}^{0}\right)+K\left(\bar{w}^{k+1}-\underline{w}^{0}\right)>0 \text { in } B(\tilde{x}, \rho), \\
\bar{w}^{k+1}-\underline{w}^{0}>0 \text { in } \partial B(\tilde{x}, \rho),
\end{array}\right.
$$

such that we have that $\bar{w}^{k+1}-\underline{w}^{0}>0$ in $\overline{B(\tilde{x}, \rho)}$. The remainder of the proof is a standard monotone iteration argument, just as in the proof of Lemma 5.3 of [23]. We observe that

$$
\left\{\begin{array}{l}
-D \mathcal{L}\left(\bar{w}^{1}-\bar{w}^{0}\right)+K\left(\bar{w}^{1}-\bar{w}^{0}\right)=K \bar{w}^{0}+F\left(x, \bar{w}^{0}\right)-K \bar{w}^{0}<0 \text { in } \Omega, \\
\nabla\left[\bar{w}^{1}-\bar{w}^{0}\right] \cdot v=0 \text { on } \partial \Omega
\end{array}\right.
$$

Thus, by the strong maximum principle, we have $\bar{w}^{1}<\bar{w}^{0}$.

Similarly, if $\bar{w}^{k}<\bar{w}^{k-1}$, then

$$
\left\{\begin{array}{l}
-D \mathcal{L}\left(\bar{w}^{k+1}-\bar{w}^{k}\right)+K\left(\bar{w}^{k+1}-\bar{w}^{k}\right)= \\
K \bar{w}^{k}+F\left(x, \bar{w}^{k}\right)-K \bar{w}^{k-1}-F\left(x, \bar{w}^{k-1}\right)<0 \text { in } \Omega, \\
\nabla\left[\bar{w}^{k+1}-\bar{w}^{k}\right] \cdot v=0 \text { on } \partial \Omega .
\end{array}\right.
$$

By induction, the sequence $\left\{\bar{w}^{k}\right\}$ is decreasing, and it is bounded below by $\max \left\{0, \underline{w}^{0}(x)\right\}$, so by standard elliptic theory, it converges to a nonnegative nontrivial solution of Equation (3) as $k \rightarrow \infty$. Since by Lemma 3 the nontrivial nonnegative solution of Equation (3) is unique, it coincides with the one constructed as the limit of the sequence $\left\{\bar{w}^{k}\right\}$.

Define $\bar{W}^{0}=\bar{w}^{0}$ and $\bar{W}^{k+1}=\bar{W}^{k}+F\left(x, \bar{W}^{k}\right)$ in $\Omega$. Following the proof of Lemmas 5.6 and 5.7 in [23], we can prove the following result.

Lemma 7. Suppose that Equation (8) holds. For every $k$, we have

$$
\underline{w}^{0}<\bar{W}^{k+1}<\bar{W}^{k}
$$

Then, $\bar{W}^{k}$ converges locally uniformly to $W^{\infty}$ as $k \rightarrow \infty$, with

$$
W^{\infty}=(U(x), V(x)) \text { in } \Omega_{0}, \text { and } W^{\infty}(x)=0 \text { in } \Omega \backslash \Omega_{0},
$$

where $\Omega_{0}$ is given by Equation (11).

Proof. Observe that, by Equation (15), the function $\underline{w}^{0}$ is a subsolution of the kinetic system. Repeating the proof of Lemma 5.6 in [23], or following the arguments leading to the monotonicity of the proof of Lemma 6 above, we have that the sequence $\left\{\bar{W}^{k}\right\}$ is monotone decreasing and bounded below by $\underline{w}^{0}$. Therefore, $\bar{W}^{k} \rightarrow W^{\infty}$ pointwise, which satisfies $F\left(x, W^{\infty}\right)=0$, i.e. a nonnegative equilibrium of the kinetic system. Therefore, if at some $x \in \Omega$ we have that $(s(x)+a(x)) e(x)-r(x) s(x) \geq 0$, then $W^{\infty}(x)=0$. On the other hand, $W^{\infty}(x) \geq \underline{w}^{0}(x)>0$ in $B(\tilde{x}, \rho) \subset \Omega_{0}$ for $x \in \Omega_{0}$. Since $x$ is arbitrary and the sequence does not depend on $\underline{w}^{0}$, we obtain that $W^{\infty}(x)=(U(x), V(x))$, the unique positive kinetic equilibrium, whenever $x \in \Omega_{0}$. Particularly, $W^{\infty}$ is continuous. Using Theorem 5.8 of [23], we obtain that the convergence is uniform in any compact set of $\Omega$.

Lemma 8. For each $k$, as $d_{1}, d_{2} \rightarrow 0$, we have that $\bar{w}^{k}$ converges to $\bar{W}^{k}$ uniformly in $\bar{\Omega}$.

The proof of this result is the same as the one of Lemma 5.5 in [23]. 
Proof of Theorem 7. Using a diagonal argument, and Lemma (8), the unique positive solution $w$ of Equation (3) converges to $W_{\infty}$ as $d_{1}, d_{2} \rightarrow \infty$.

\section{The Case of $d_{1}$ and $d_{2}$ Large}

We will start by giving a proof of a result that is well known as a "folk theorem." It is stated in slightly more generality than is needed for the specific application. For $i=1, \ldots, N$, let $L^{i}$ denote the operator

$$
L^{i} u=\nabla \cdot \mu_{i}(x)\left[\nabla u-u \nabla \alpha_{i}(x)\right] \text { for } x \in \Omega
$$

with no-flux boundary conditions

$$
\left[\nabla u-u \nabla \alpha_{i}\right] \cdot v=0 \quad \text { for } \quad x \in \partial \Omega \text {. }
$$

Assume that $\mu_{i}(x) \geq \mu_{0}>0$ on $\bar{\Omega}$ for all $i$. Let $A=\left(a_{i j}(x)\right)$ be an $N \times N$ irreducible matrix with $a_{i j} \geq 0$ if $i \neq j$. Consider the eigenvalue problem

$$
d_{i} L^{i} \varphi_{i}+\sum_{j=1}^{N} a_{i j} \varphi_{j}=\lambda \varphi_{j}, \quad i=1 \ldots N
$$

where $d_{i}>0$ for all $i$ and $\varphi_{i}$ satisfies the boundary condition expressed in Equation (17) for each $i$. Note that, if we let $\Phi_{i}=\exp \left(-\alpha_{i}(x)\right) \varphi_{i}$, then $\Phi_{i}$ satisfies Neumann boundary conditions such that the system expressed in Equation (18) rewritten in terms of the variables $\Phi_{i}$ is still cooperative. Because of the classical boundary conditions, the usual results on elliptic regularity and on maximum principles for cooperative systems from $[15,23]$ can be applied to the system for the $\Phi_{i}$ values, such that the system and hence Equation (18) will have a principal eigenvalue under suitable conditions on the domain $\Omega$ and the coefficients. This idea has been used in models for single populations without an age structure or competing pairs of such populations-see, for example, [2,24].

Furthermore, we have $L^{i}\left(\exp \left(\alpha_{i}(x)\right)=0\right.$ such that the principal eigenvalue of $L^{i}$ is zero, and the eigenfunction is a multiple of $\exp \left(\alpha_{i}\right)$. Let $\bar{A}$ be the matrix defined by

$$
\bar{A}_{i j}:=\frac{\int_{\Omega} a_{i j} \exp \left(\alpha_{i}\right) d x}{\int_{\Omega} \exp \left(\alpha_{i}\right) d x} .
$$

Denote the principal eigenvalue of Equation (18) as $\lambda_{1}(\vec{d})$ where $\vec{d}=\left(d_{1}, \ldots, d_{N}\right)$. Denote the principal eigenvalue of $\bar{A}$ as $\bar{\Lambda}$.

Lemma 9. Suppose that, for some $\gamma \in(0,1)$, the coefficients of Equation (18) satisfy $\alpha \in C^{2, \gamma}(\bar{\Omega}), \mu \in$ $C^{1, \gamma}(\bar{\Omega})$, and $a_{i j} \in C^{\gamma}(\bar{\Omega})$ for $i, j=1 \ldots N$, and that $\partial \Omega$ is of class $C^{2, \gamma}$. Suppose further that $\bar{A}$ is irreducible. If $\min \left\{d_{i}: i=1, \ldots N\right\} \rightarrow \infty$, then $\lambda_{1}(\vec{d}) \rightarrow \bar{\Lambda}$.

Proof. Choose any sequence $\vec{d}_{n}=\left(d_{1 n}, \ldots d_{N n}\right)$ such that $\min \left\{d_{i n}: i=1, \ldots N\right\} \rightarrow \infty$. Choose any subsequence, then renumber it as $\vec{d}_{n}$. Let $\lambda_{n}$ be the principal eigenvalue of Equation (18) corresponding to $\vec{d}_{n}$ and let $\varphi_{i n}(x)>0$ be the $i$ th component of the eigenvector, where the eigenvector is normalized by $\max \left\{\varphi_{\text {in }}(x): x \in \bar{\Omega}, i=1, \ldots, N\right\}=1$. Integrating the $i$ th equation of Equation (18) over $\Omega$ and summing over $i$ yields

$$
\lambda_{n} \int_{\Omega} \sum_{i=1}^{N} \varphi_{i n}(x) d x=\int_{\Omega} \sum_{i, j=1}^{N} a_{i j}(x) \varphi_{j n}(x) d x \leq A_{1} \int_{\Omega} \sum_{i=1}^{N} \varphi_{i n}(x) d x
$$


where $A_{1}$ is a constant, depending only on $A$. It follows that $\lambda_{n}$ is uniformly bounded from above. Similarly, $\lambda_{n}$ is uniformly bounded from below. Thus, any subsequence of $\lambda_{n}$ itself has a convergent subsequence. It then follows from dividing the $i$ th equation of Equation (18) by $d_{i n}$ that $L^{i} \varphi_{i n}$ is uniformly bounded, and $L_{i} \varphi_{i n} \rightarrow 0$ as $n \rightarrow \infty$. By elliptic regularity, the sequence $\varphi_{i n}$ is uniformly bounded in $W^{2, p}(\Omega)$ for any $p<\infty$, then by Sobolev embedding, it has a subsequence that is convergent in $C^{1}(\bar{\Omega})$ and weakly convergent in $W^{2, p}(\Omega)$. This will be true for any $i$. Taking a further subsequence if necessary and renumbering again, we obtain a sequence where $\lambda_{n} \rightarrow \lambda^{*}$ for some $\lambda^{*}$ and $\varphi_{\text {in }} \rightarrow \varphi_{i}^{*}$ for all $i$, with $L_{i} \varphi_{i}^{*}=0$. We then must have $\varphi_{i}^{*}=c_{i} \exp \left(\alpha_{i}\right)$ for some nonnegative constant $c_{i}$, and with $\max \left\{\varphi_{i}^{*}(x): x \in \bar{\Omega}, i=1 \ldots N\right\}=1$. Integrating Equation (18) over $\Omega$ and using the no-flux boundary conditions gives

$$
\sum_{j=1}^{N}\left[\int_{\Omega} a_{i j}(x) \varphi_{j}^{*}(x) d x\right]=\lambda^{*} \int_{\Omega} \varphi_{i}^{*}, \quad i=1 \ldots N,
$$

such that

$$
\sum_{j=1}^{N}\left[\frac{\int_{\Omega} a_{i j}(x) \exp \left(\alpha_{j}(x)\right) d x}{\int_{\Omega} \exp \left(\alpha_{i}(x)\right) d x}\right] c_{j}=\lambda^{*} c_{i}, \quad i=1 \ldots N .
$$

It follows that $\left(c_{1}, \ldots, c_{N}\right)$ must be a nontrivial nonnegative eigenvector of $\bar{A}$ with the normalization prescribed by $\max \left\{c_{i} \exp \left(\alpha_{i}(x)\right): x \in \bar{\Omega}, i=1, \ldots N\right\}=1$. These last conditions uniquely determine the limits of the subsequence of the original subsequence $\left\{\lambda\left(d_{n}\right), \vec{\varphi}_{n}\right\}$. Since every subsequence of the original sequence $\left\{\lambda\left(d_{n}\right), \vec{\varphi}_{n}\right\}$ has a subsequence converging to the values determined by Equation (21), the same must be true for the original sequence. Since the original sequence of values $\left\{\vec{d}_{n}\right\}$ could be any increasing sequence that approaches infinity as $n \rightarrow \infty$, the conclusion of the lemma follows.

In the specific system expressed in Equation (1) that we consider, $L^{i}=\Delta$, such that $\alpha_{i}$ and $\mu_{i}$ are constants. In that case, we have $\bar{A}_{i j}=\bar{a}_{i j}$, where $\bar{a}_{i j}$ is the average of $a_{i j}$ over $\Omega$. Denote the averages of the coefficients in Equation (1) by $\bar{r}, \bar{s}$, etc. Calculations analogous to those in Equation (5), Equation (6), and the related discussion then yield the following:

Corollary 1. Suppose that the hypotheses of Lemma 9 are satisfied. There exists a $D>0$ such that if

$$
\bar{e}(\bar{s}+\bar{a})-\bar{r} \bar{s}<0,
$$

the principal eigenvalue $\lambda_{1}$ of Equation (2) is positive for all $d_{1}, d_{2}>D$, while if

$$
\bar{e}(\bar{s}+\bar{a})-\bar{r} \bar{s}>0,
$$

the principal eigenvalue is nonpositive.

Remark 2. In the ODE system corresponding to Equation (1) with coefficients averaged over $\Omega$, one can compute $R_{0}$ as $\bar{r} \bar{s} /[\bar{e}(\bar{s}+\bar{a})]$ via the methods of [25]. The first inequality in Corollary 1 is equivalent to $R_{0}>1$, while the second is equivalent to $R_{0}<1$. By writing $R_{0}=[\bar{r} /(\bar{s}+\bar{a})][\bar{s} / \bar{e}]$, we can interpret the condition for persistence as saying that the products of the ratios of the growth terms over the loss terms for adults and juveniles should be greater than 1 for persistence.

\section{General Diffusion Rates}

Case1: Persistence or extinction for all diffusion rates 
Proposition 2. If

$$
\int_{\Omega} \sqrt{r s} d x-\frac{1}{2} \int_{\Omega}(s+a+e) d x>0
$$

then $\lambda_{1}>0$ for all positive diffusion rates.

If

$$
\min _{x \in \bar{\Omega}}\left[4(s(x)+a(x)) e(x)-(r(x)+s(x))^{2}\right]>0
$$

then $\lambda_{1}<0$ for all positive diffusion rates.

Proof. If we divide the first equation in Equation (2) by $\varphi$ and integrate over $\Omega$, using Green's formula to integrate the term $\Delta \varphi / \varphi$, we obtain the inequality

$$
|\Omega| \lambda_{1} \geq \int_{\Omega} r\left(\frac{\psi}{\varphi}\right) d x-\int_{\Omega}(s+a) d x
$$

Similarly, if we divide the second equation by $\psi$ and integrate we obtain

$$
|\Omega| \lambda_{1} \geq \int_{\Omega} s\left(\frac{\varphi}{\psi}\right) d x-\int_{\Omega} e d x
$$

If we add Equations (24) and (25) and divide by 2, we obtain

$$
\lambda_{1} \geq \frac{1}{2|\Omega|}\left(\int_{\Omega}\left[r\left(\frac{\psi}{\varphi}\right)+s\left(\frac{\varphi}{\psi}\right)\right] d x-\int_{\Omega}(s+a+e) d x\right) .
$$

By Cauchy's inequality, $r z+s z^{-1} \geq 2 \sqrt{r s}$ for all $z>0$, so from Equation (26) we obtain

$$
\lambda_{1} \geq \frac{1}{|\Omega|}\left[\int_{\Omega} \sqrt{r s} d x-\frac{1}{2} \int_{\Omega}(s+a+e) d x\right]
$$

Thus, $\lambda_{1}>0$ if Equation (22) holds, so the first part of Proposition 2 holds. Going in the other direction, if we multiply the first equation of Equation (2) by $\varphi$ and integrate, using integration by parts on the $\varphi \Delta \varphi$ term, and similarly multiply the second equation by $\psi$ and integrate, and then add the results, we get

$$
\left.\lambda_{1} \int_{\Omega}\left(\varphi^{2}+\psi^{2}\right) d x \leq \int_{\Omega}\left[-(s+a) \varphi^{2}+(r+s) \varphi \psi-e \psi^{2}\right) d x\right] .
$$

The integrand on the right side of Equation (28) is a quadratic form in $\varphi$ and $\psi$, which will be negative definite if

$$
4(s+a) e>(r+s)^{2},
$$

so $\lambda_{1}<0$ if Equation (23) holds, which proves the second part of Proposition 2.

Remarks: Note that the first integral in Equation (22) is what appears in the formula for the Bhattacharyya coefficient $[26,27]$, which is used to compare how well probability distributions match each other. Specifically, if two probability distributions $P$ and $Q$ have probability density functions $p(x)$ and $q(x)$ for $x \in U \subset \mathbb{R}^{n}$, the Bhattacharyya coefficient is

$$
B C(P, Q)=\int_{U} \sqrt{p(x) q(x)} d x .
$$

For any $P$ and $Q, 0 \leq B C(P, Q) \leq 1$. If $B C(P, Q)=1$, then $P$ and $Q$ are the same, that is, $p=q$ a.e. If $B C(P, Q)=0$, then the supports of $p$ and $q$ are disjoint. If we write $r(x)=r_{0} \rho(x)$ and $s(x)=s_{0} \sigma(x)$ 
such that $\int_{\Omega} \rho(x)=\int_{\Omega} \sigma(x)=1$, then we can compute $r_{0}=\bar{r}|\Omega|$ and $s_{0}=\bar{s}|\Omega|$. We can treat $\rho$ and $\sigma$ as if they were probability density functions for distributions $R$ and $S$. We then have

$$
\int_{\Omega} \sqrt{r s} d x=|\Omega| \sqrt{\bar{r} \bar{s}} B C(R, S) .
$$

The maximum of $B C(R, S)$ is 1, corresponding to the case where $r$ and $s$ are multiples of each other, and the minimum is 0 , corresponding to the case where the supports of $r$ and $s$ are disjoint. Thus, the degree to which $\rho$ and $\sigma$ match each other has a strong impact on the estimate for $\lambda$ in Equation (27).

Using Equation (30) and the fact that $B C(R, S) \leq 1$ in Equation (22) shows that Equation (22) implies $2 \sqrt{\bar{r} \bar{s}}>[(\bar{s}+\bar{a})+\bar{e}]$. Squaring both sides and using Cauchy's inequality implies $\bar{e}(\bar{s}+\bar{a})-$ $\bar{r} \bar{s}<0$ as in the first case of Corollary 1. Similarly, if Equation (22) holds, then $2 \sqrt{r(x) s(x)}>$ $(s(x)+a(x))+e(x)$ for some $x \in \Omega$, and it then follows in the same way that the inequality in the first case of Proposition 1 holds. If Equation (23) holds, then Equation (29) holds, and then by Cauchy's inequality the second case of Proposition 1 holds. Thus, the conditions expressed in Equation (22) and Equation (23) in Proposition 2, which imply $\lambda_{1}>0$ or $\lambda_{1}<0$ for all diffusion rates, also imply some of the corresponding conditions we have obtained for either large or small diffusion rates.

In the situation where the spatial distributions of habitat quality $r$ for reproduction by adults and $s$ for survival and maturation of juveniles into adults are perfectly correlated, such that $r(x)=r_{1} s(x)$ for some constant $r_{1}$, the eigenvalue problem expressed in Equation (2) can be rewritten as a weighted symmetric eigenvalue problem by multiplying the second equation in Equation (2) by $r_{1}$, which yields

$$
\begin{aligned}
& d_{1} \Delta \varphi(x)-(s(x)+a(x)) \varphi+r(x) \psi=\lambda \varphi \\
& d_{2} r_{1} \Delta \psi+r(x) \varphi-r_{1} e(x) \psi=\lambda r_{1} \psi .
\end{aligned}
$$

The principal eigenvalue for Equation (31) has a variational characterization of $\lambda_{1}$ as

$$
\lambda_{1}=\max _{\varphi, \psi \in W^{1,2}(\Omega)} \frac{\int_{\Omega}\left(-d_{1}|\nabla \varphi|^{2}-d_{2} r_{1}|\nabla \psi|^{2}-(s+a) \varphi^{2}+2 r \varphi \psi-r_{1} e \psi^{2}\right) d x}{\int_{\Omega}\left(\varphi^{2}+r_{1} \psi^{2}\right) d x} .
$$

It follows in that case that $\lambda_{1}$ is decreasing in both $d_{1}$ and $d_{2}$, such that slower diffusion is advantageous.

Case 2: Asymptotic behavior for large reproductive rates

Suppose that $r(x)=n r_{0}(x)$ and that $s(x) r_{0}(x)>0$ for $x \in \Omega_{0}$ with $\Omega_{0} \neq \varnothing$; therefore, there is a region where both the adult reproduction rate and the juvenile maturation rate are positive. The factor $n$ scales the reproductive rate of adults in regions where $r_{0}(x)>0$. For any fixed diffusion rates, it turns out that, for sufficiently large values of the scaling coefficient $n$, the principal eigenvalue of Equation (2) is positive, so the system expressed in Equation (1) is persistent. We will characterize the asymptotic behavior of the principal eigenvalue as $n \rightarrow \infty$. If we make the further assumption that $g \equiv c \equiv 0$, then the system expressed in Equation (1) is cooperative, so for an $n$ large enough that the principal eigenvalue of Equation (2) is positive, Equation (1) has a unique positive equilibrium, and we will characterize the behavior of that equilibrium as $n \rightarrow \infty$ as well in that case.

Let $\lambda_{1}^{n}$ denote the principal eigenvalue for Equation (2) with $r(x)=n r_{0}(x)$. Observe that, since $s(x) r_{0}(x)>0$ for $x \in \Omega_{0}$ and $\Omega_{0} \neq \varnothing$, Proposition 2 implies that $\lambda_{1}^{n}>0$ for $n$ sufficiently large, and in fact by Equation (27), $\lambda_{1}^{n} \rightarrow \infty$ as $n \rightarrow \infty$. The following proposition states the asymptotic behavior of $\lambda_{1}^{n}$ as $n \rightarrow \infty$.

Proposition 3. If $r(x)=n r_{0}(x)$ and $s(x) r_{0}(x)>0$ for $x \in \Omega_{0}$ with $\Omega_{0} \neq \varnothing$, then

$$
\lim _{n \rightarrow \infty} \frac{\lambda_{1}^{n}}{\sqrt{n}} \rightarrow \max _{x \in \bar{\Omega}}\left(\sqrt{r_{0}(x) s(x)}\right) .
$$


Proof. We start by noting that, if $\lambda_{1}^{n}$, then $\left(\varphi_{n}, \psi_{n}\right)$ are the principal eigenvalue and corresponding eigenfunction of Equation (2) for $r(x)=n r_{0}(x)$; therefore, $\lambda_{1}^{n} / \sqrt{n}, \widehat{\varphi}_{n}=\varphi_{n}$, and $\widehat{\psi}_{n}=\sqrt{n} \psi_{n}$ are the principal eigenvalue and eigenfunction of the following problem:

$$
\left\{\begin{aligned}
\frac{d_{1}}{\sqrt{n}} \Delta \widehat{\varphi}-\frac{(s(x)+a(x))}{\sqrt{n}} \widehat{\varphi}+r_{0}(x) \widehat{\psi} & =\widehat{\lambda} \widehat{\varphi} \text { in } \Omega, \\
\frac{d_{2}}{\sqrt{n}} \Delta \widehat{\psi}-\frac{e(x)}{\sqrt{n}} \widehat{\psi}+s(x) \widehat{\varphi} & =\widehat{\lambda} \widehat{\psi} \text { in } \Omega, \\
\nabla \widehat{\varphi} \cdot v=\nabla \widehat{\psi} \cdot v & =0 \text { on } \partial \Omega .
\end{aligned}\right.
$$

Considering the elliptic operators $L_{1} u=d_{1} \Delta u-(s(x)-a(x)) u$ and $L_{2} v=d_{2} \Delta v-e(x) v$, $D=\operatorname{diag}\left(\frac{1}{\sqrt{n}}, \frac{1}{\sqrt{n}}\right)$ and $\mathcal{L}=\operatorname{diag}\left(L_{1}, L_{2}\right)$, the system expressed in Equation (33) satisfies the hypothesis of Theorem 1.4 of [23]. Thus, $n \rightarrow \infty$

$$
\frac{\lambda_{1}^{n}}{\sqrt{n}} \rightarrow \max _{x \in \bar{\Omega}} \lambda(A(x))
$$

where

$$
A(x)=\left(\begin{array}{cc}
0 & r_{0}(x) \\
s(x) & 0
\end{array}\right),
$$

which has eigenvalues $\pm \sqrt{r_{0}(x) s(x)}$, from whence the result follows.

In the case where $g \equiv c \equiv 0$ such that Equation (1) is cooperative, Lemma 3 implies that Equation (1) has a unique positive equilibrium if the principal eigenvalue of Equation (2) is positive. The next result states the asymptotic behavior of the unique positive equilibrium of Equation (1) for $n$ large in that case.

Proposition 4. Suppose the hypotheses of Proposition 3 are satisfied and that $g \equiv c \equiv 0$. Let $\left(u_{n}, v_{n}\right)$ be the unique positive solution of Equation (3). Then $n^{-\frac{2}{3}}\left(u_{n}, v_{n}\right) \rightarrow\left(U^{\infty}, V^{\infty}\right)$ uniformly in $\bar{\Omega}$ where

$$
\begin{aligned}
& U^{\infty}(x)=\frac{r_{0}(x)^{\frac{2}{3}}}{b(x)^{\frac{2}{3}}} \frac{s(x)^{\frac{1}{3}}}{f(x)^{\frac{1}{3}}}, V^{\infty}(x)=\frac{s(x)^{\frac{2}{3}}}{f(x)^{\frac{2}{3}}} \frac{r_{0}(x)^{\frac{1}{3}}}{b(x)^{\frac{1}{3}}} \text { when } s(x) r_{0}(x)>0 \\
& U^{\infty}(x)=0, V^{\infty}(x)=0 \text { when } s(x) r_{0}(x)=0 .
\end{aligned}
$$

Proof. To prove this result, we use a different scaling. After some simple computations, we find that

$$
\left(w_{n}, z_{n}\right)=\left(u_{n} n^{-\frac{2}{3}}, v_{n} n^{-\frac{1}{3}}\right),
$$

is the unique positive solution of the scaled system

$$
\left\{\begin{array}{l}
n^{-\frac{2}{3}}\left[d_{1} \Delta w-(s(x)+a(x)) w\right]+r_{0}(x) z-b(x) w^{2}=0 \text { in } \Omega \\
n^{-\frac{1}{3}}\left[d_{2} \Delta z-e(x) z\right]+s(x) w-f(x) z^{2}=0 \text { in } \Omega \\
\nabla w \cdot v=\nabla z \cdot v=0 \text { on } \partial \Omega .
\end{array}\right.
$$

We set the operators

$$
\begin{gathered}
L_{1} w=d_{1} \Delta w-(s(x)+a(x)) w \text { and } L_{2} z=d_{2} \Delta z-e(x) z, \\
D=\operatorname{diag}\left(n^{-\frac{2}{3}}, n^{-\frac{1}{3}}\right) \text { and } \mathcal{L}=\left(L_{1}, L_{2}\right), \text { and } \\
F(x, w, z)=\left(r_{0}(x) z-b(x) w^{2}, s(x) w-f(x) z^{2}\right) .
\end{gathered}
$$


To prove the proposition, we can follow the same steps as in the proof of Theorem 1 . Indeed, the principal eigenvalue of the linearization around $(0,0)$ of the associated kinetic system of Equation (35) is $\sqrt{s(x) r_{0}(x)}$, and when it is positive, the kinetic equilibrium is given by the right hand side of Equation (34). This concludes the proof.

\section{Conclusions}

The most fundamental conclusion from our analysis is that reaction-diffusion models for populations with a stage structure in spatially heterogeneous environments do not necessarily predict that slower diffusion is advantageous for persistence. This is in contrast to the case where populations are structured only by spatial location, where a version of the reduction principle [6] applies; in a competition between otherwise identical populations with different diffusion rates, the prediction is that "the slower diffuser wins" [4,5]. The mechanism underlying this observation is that, in our structured model, the regions where it is possible for adults to produce offspring may be separated from those where juveniles can survive and mature into adults. The conditions we find that imply persistence generally require that the product $r(x) s(x)$ of the reproductive rate of adults and the maturation rate of juveniles be sufficiently large relative to their death rates. For slow diffusion, the condition for persistence is that $r(x) s(x)>e(x)(s(x)+(a(x))$ at some point $x \in \Omega$. For fast diffusion, it is $\bar{r} \bar{s}>\bar{e}(\bar{s}+\bar{a})$ where $\bar{r}, \bar{s}, \bar{e}$, and $\bar{a}$ are the spatial averages of those quantities. If the spatial distributions of $r$ and $s$ are closely correlated and are large in a few places but small in most, such that the maximum of $r s$ is large but the averages $\bar{r}$ and $\bar{s}$ are small, the condition for persistence with slow diffusion may be satisfied, while the condition with fast diffusion may fail. In that type of environment, slow diffusion is clearly favored. Furthermore, if $r$ and $s$ are perfectly correlated in the sense that they are multiples of each other, the principal eigenvalue determining the growth rate of the population at low density is decreasing with respect to the diffusion rates, as in the case of unstructured populations in heterogeneous environments. On the other hand, if both $r$ and $s$ are large on some regions but very small outside of them, and the regions where they are large are disjoint (that is, separated from each other), then the product $r$ s could be small everywhere, but the averages $\bar{r}$ and $\bar{s}$ could be large. In that case, the condition for persistence with small diffusion may fail, but the condition with fast diffusion may be satisfied, such that fast diffusion is favored.

We found that a sufficient condition for persistence for all diffusion rates is

$$
\int_{\Omega} \sqrt{r s} d x-\frac{1}{2} \int_{\Omega}(s+a+e) d x>0 .
$$

The first term can be written as $\sqrt{\bar{r} \bar{s}}|\Omega| B C(r(x) / \bar{r}, s(x) / \bar{s})$, where $B C$ denotes the Bhattacharrya coefficient (see [26,27]), which measures how closely probability densities match each other. For distributions that are equal to each other, $B C=1$, but for distributions that are mutually exclusive in the sense that the regions where they are positive do not intersect, $B C=0$. This observation again shows that the degree to which the spatial distributions of $r$ and $s$ match each other is significant in determining the predictions of the model expressed in Equation (1).

Finally, we found that, if we scale the adult reproductive rate as $r(x)=n r_{0}(x)$ and there is some overlap between the distributions of $r$ and $s$ such that $s(x) r_{0}(x)>0$ on some subset of $\Omega$ with positive measure, then for any fixed diffusion rates the system expressed in Equation (1) will be persistent if $n$ is sufficiently large. This means a population with any diffusion rates can persist if there is even a modest overlap between the regions where adults can reproduce and where juveniles can mature, provided that the reproductive rate of adults is sufficiently large. We characterized the asymptotic behavior as $n \rightarrow \infty$ of the principal eigenvalue of Equation (2). In the cooperative case where $g \equiv c \equiv 0$, the system expressed in Equation (1) will have a unique positive equilibrium if it is persistent, and in that case we also characterized the asymptotic behavior as $n \rightarrow \infty$ of the equilibrium.

There are several directions for further research on the general topic of this paper. It would be of interest to take the approach of [4] and consider the competition between two stage structured 
populations described by systems such as Equation (1) that differ only in their diffusion rates. That would be somewhat challenging because it would involve systems of four equations, but at least in the cooperative case where $c=g=0$ the general theory of monotone dynamical systems as in [28] and some of the ideas and methods of [10] would apply. It would also be interesting to consider models with an explicit age structure, as introduced in [11] and studied in [12,13]. Finally, it would be interesting albeit challenging to consider the case of time-periodic environments with spatial heterogeneity. Temporal variation alone is sufficient to cause faster diffusion to be favored in such environments in some cases (see [29]), but even without a stage structure, the time-dependent case is challenging, and there are many open questions.

Author Contributions: Conceptualization, R.S.C. C.C., and S.M.;mathematical analysis, R.S.C., C.C. and S.M.; writing-original draft preparation, C.C. and S.M.; writing-review and editing, R.S.C., C.C., and S.M.; funding acquisition, R.S.C., C.C. and S.M. All authors have read and agreed to the published version of the manuscript.

Funding: This research received external funding as noted in the subsequent Acknowledgments .

Acknowledgments: R.S.C. and C.C. are supported in part by NSF Awards DMS 15-14792 and 18-53478. S.M. is supported by CONICYT + PIA/Concurso de Apoyo a Centros Científicos y Tecnológicos de Excelencia con Financiamiento Basal AFB170001.

Conflicts of Interest: The authors declare no conflict of interest.

\section{Appendix A}

In [23], the authors considered the equilibria and dynamics of the system

$$
\left\{\begin{array}{l}
\frac{\partial u}{\partial t}=D \mathcal{L} u+F(x, u) \text { in } \Omega \times(0, \infty), \\
\mathcal{B} u=0 \text { on } \partial \Omega \times(0, \infty)
\end{array}\right.
$$

where $u=\left(u_{1}, \ldots, u_{n}\right)^{T}$ is a vector of smooth functions, $\Omega$ is a bounded domain in $\mathbb{R}^{N}$ with smooth boundary, $u=\left(u_{1}(x), \ldots u_{n}\right)^{T}$ is a vector of smooth functions, $D=\operatorname{diag}\left(d_{1}, \ldots d_{n}\right)$ is a diagonal matrix of positive constants, $\mathcal{L}=\operatorname{diag}\left(L^{1}, \ldots L^{n}\right)$ is a diagonal matrix of second order uniformly strongly elliptic operators of the form

$$
L^{i}=\sum_{j, k=1}^{N} \alpha_{j k}^{i} \frac{\partial^{2}}{\partial x_{j} \partial x_{k}}+\sum_{j=1}^{N} \beta_{j}^{i} \frac{\partial}{\partial x_{j}}+\gamma^{i}
$$

with smooth coefficients, and $\mathcal{B}=\left(B_{1}, \ldots B_{n}\right)$, where for each $i, B_{i}$ defines a Dirichlet, Neumann, or Robin boundary condition. (They include Neumann as a case of Robin.) They also considered the associated linearized problem, which they wrote as

$$
\left\{\begin{array}{l}
D \mathcal{L} \phi+A u \phi=-\lambda \phi \text { in } \Omega, \\
B \phi=0 \text { on } \partial \Omega
\end{array}\right.
$$

where $A=\left(a_{i j}\right)$ is an $n \times n$ matrix of smooth functions with $a_{i j} \geq 0$ for $i \neq j$, and $\phi=$ $\left(\phi_{1}(x), \ldots \phi_{n}(x)\right)^{T}$ is a vector of smooth functions.

Note that, in our notation, we use the opposite sign convention to the one used in [23], such that what they denote as $-\lambda$, we denote as $\lambda$.

For details on what the specific smoothness assumptions require, see [23]. Under those assumptions, by the Perron-Frobenius theorem, for each $x \in \Omega$, the matrix $A$ has a principal eigenvalue, which in our notation we denote as $\Lambda(x)$. The first major result of [23] is their Theorem 1.4, which can be stated as follows: 
Theorem A1. (Theorem 1.4 of [23]) The principal eigenvalue $\lambda_{1}$ of the system expressed in Equation (A2) with Dirichlet, Neumann, or Robin boundary conditions satisfies

$$
\lim _{\max \left\{d_{1}, \ldots, d_{n}\right\} \rightarrow 0} \lambda_{1}=-\max _{x \in \bar{\Omega}} \Lambda(x) .
$$

Except for the adjustments needed for our different notation, that theorem applies directly to our system in all cases.

The second major result of [23] gives conditions under which the system expressed in Equation (A1) with small diffusion rates has the same dynamics as the kinetic system

$$
\frac{d U_{i}}{d t}=F_{i}\left(x, U_{1}, \ldots, U_{n}\right) \text { for } i=1, \ldots, n .
$$

The conditions can be stated as follows, noting that we have replaced the A used in [23] with L to avoid any confusion with equation numbers in this Appendix:

(L1) $\partial F_{i} / \partial U_{j} \geq 0$ (i.e. the systems expressed in Equations (A1) and (A3) are cooperative).

(L2) For each $x_{0} \in \bar{\Omega}$, the system expressed in Equation (A3) has a unique positive equilibrium $\alpha\left(x_{0}\right)$, which is globally asymptotically stable among positive solutions and is locally linearly stable, and $\alpha(x)$ depends continuously on $x$.

(L3) There is a $\delta_{0}>0$ such that, for $j=1, \ldots, n, F_{j}(x, U) / U_{j}>\delta_{0}$ for all $x \in \bar{\Omega}$ provided $0<U_{i} \leq \delta_{0}$ for $i=1, \ldots, n$.

(L4) There is a $\delta_{0}^{\prime}, M>0$ such that for $j=1, \ldots, n, F_{j}(x, U) / U_{j}<-\delta_{0}^{\prime}$ for all $x \in \bar{\Omega}$ provided $U_{i} \geq M$ for $i=1, \ldots, n$.

The second result of [23] that we use is Proposition 5.2, which can be stated as follows:

Theorem A2. (Proposition 5.2 of [23]) For any positive steady state $w_{d}$ of Equation (A1), we have that $w \rightarrow \alpha$ uniformly in $\Omega$ as $\max \left\{d_{1}, \ldots, d_{n}\right\} \rightarrow 0$, with the $\alpha$ given in Assumption (L2).

\section{References}

1. Cantrell, R.S.; Cosner, C. Spatial Ecology via Reaction-Diffusion Equations; John Wiley and Sons: Chichester, UK, 2003.

2. Cosner, C. Reaction-diffusion-advection models for the effects and evolution of dispersal. Discret. Cont. Dyn. Syst. A 2014, 34, 1701-1745. [CrossRef]

3. He, X.; Ni, W.-M. Global dynamics of the Lotka-Volterra competition-diffusion system: Diffusion and Spatial Heterogeneity I. Commun. Pure Appl. Math. 2016, 69, 981-1014. [CrossRef]

4. Dockery, J.; Hutson, V.; Mischaikow, K.; Pernarowski, M. The evolution of slow dispersal rates: A reaction diffusion model. J. Math. Biol. 1998, 37, 61-83. [CrossRef]

5. Hastings, A. Can spatial variation alone lead to selection for dispersal? Theor. Popul. Biol. 1983, 24, 244-251. [CrossRef]

6. Altenberg, L. Resolvent positive linear operators exhibit the reduction phenomenon. Proc. Natl. Acad. Sci. USA 2012, 109, 3705-3710. [CrossRef] [PubMed]

7. Greenwood-Lee, J.; Taylor, P.D. The evolution of dispersal in spatially varying environments. Evol. Ecol. Res. 2001, 3, 649-665.

8. Brown, K.J.; Zhang, Y. On a system of reaction-diffusion equations describing a population with two age groups. J. Math. Anal. Appl. 2003, 282, 444-452. [CrossRef]

9. Cantrell, R.S.; Cosner, C.; Yu, X. Dynamics of populations with individual variation in dispersal on bounded domains. J. Biol. Dyn. 2018, 12, 288-317. [CrossRef]

10. Cantrell, R.S.; Cosner, C.; Yu, X. Populations with individual variation in dispersal in heterogeneous environments: Dynamics and competition with simply diffusing populations. Sci. China Math. 2020, 63, 441-464, arXiv:2001.03686. 
11. Gurtin, M.E. A system of equations for age-dependent population diffusion. J. Theor. Biol. 1973, 40, 389-392. [CrossRef]

12. Kunisch, K.; Schappacher, W.; Webb, G.F. Nonlinear age-dependent population dynamics with random diffusion. Comput. Math. Appl. 1985, 11, 155-173. [CrossRef]

13. Langlais, M. A nonlinear problem in age-dependent population diffusion. SIAM J. Math. Anal. 1985, 16, 510-529. [CrossRef]

14. Yan, S.; Guo, S. Dynamics of a Lotka-Volterra competition-diffusion model with stage structure and spatial heterogeneity. Discret. Contin. Dyn. Syst. B 2018, 23, 1559-1579. [CrossRef]

15. López-Gómez, J.; Molina-Meyer, M. The maximum principle for cooperative weakly coupled elliptic systems and some applications. Differ. Int. Equ. 1994, 7, 383-398.

16. Molina-Meyer, M. Global attractivity and singular perturbation for a class of nonlinear cooperative systems. J. Differ. Equ. 1996, 128, 347-378. [CrossRef]

17. Cantrell, R.S.; Cosner, C. On the effects of spatial heterogeneity on the coexistence of competing species. J. Math. Biol. 1998, 37, 103-145. [CrossRef]

18. Fernández-Rincón, S.; López-Gómez, J. A singular perturbation result in competition theory. J. Math. Anal. Appl. 2017, 445, 280-296. [CrossRef]

19. Hutson, V.; López-Gómez, J.; Mischaikow, K.; Vickers, G. Limit Behavior for a Competing Speces Problem with Diffusion. In Dynamical Systems and Applications; Agarwal, R.P., Ed.; World Sci. Publishing: Hackensack, NJ, USA, 1995; pp. 343-358.

20. Chen, S.; Shi, J. Asymptotic profiles of basic reproduction number for epidemic spreading in heterogeneous environment. arXiv 2019, arXiv:1909.10107.

21. Magal, P.; Webb, G.F, Wu,Y.-X. On the basic reproduction number of reaction-diffusion epidemic models. SIAM J. Appl. Math. 2019, 79, 284-304. [CrossRef]

22. Hei, L.J.; Wu, J.H. Existence and stability of positive solutions for an elliptic cooperative system. Acta Math. Sin. Engl. Ser. 2005, 21, 1113-1120. [CrossRef]

23. Lam, K.-Y.; Lou, Y. Asymptotic behavior of the principal eigenvalue for cooperative elliptic systems and applications. J. Dyn. Differ. Equ. 2016, 29, 29-48. [CrossRef]

24. Cantrell, R.S.; Cosner, C.; Lou, Y. Evolution of dispersal and the ideal free distribution. Math. Biosci. Eng. 2010, 7, 17-36. [PubMed]

25. Van den Driessche, P.; Watmough, J. Reproduction numbers and sub-threshold endemic equilibria for compartmental models of disease transmission. Math. Biosci. 2002, 108, 29-48. [CrossRef]

26. Bhattacharyya, A. On a measure of divergence between two statistical populations defined by their probability distribution. Bull. Calcutta Math. Soc. 1943, 35, 99-110.

27. Derpanis, K.G. The Bhattacharyya measure. Mendeley Comput. 2008, 1, 1990-1992.

28. Smith, H. Monotone Dynamical Systems. An Introduction to the Theory of Competitive and Cooperative Systems; Mathematical Surveys and Monographs, 41; American Mathematical Society: Providence, RI, USA, 1995.

29. Hutson, V.; Mischaikow, K.; Polácik, P. The evolution of dispersal rates in a heterogeneous time-periodic environment. J. Math. Biol. 2001, 43, 501-533. [CrossRef]

(C) 2020 by the authors. Licensee MDPI, Basel, Switzerland. This article is an open access article distributed under the terms and conditions of the Creative Commons Attribution (CC BY) license (http://creativecommons.org/licenses/by/4.0/). 